山्ل FRANÇAISE

$>\mathrm{DE}$

필 PÉDAGOGIE

\section{Revue française de pédagogie}

Recherches en éducation

192 | juillet-août-septembre 2015

Face aux mutations des marchés de l'emploi, quelles politiques de formation?

\title{
La professionnalisation des formations initiales : une solution aux mutations du marché de l'emploi pour les jeunes?
}

The vocationalization of initial training in higher education: a solution to changes in the labor market for young people?

Philippe Lemistre

\section{OpenEdition}

Journals

Édition électronique

URL : http://journals.openedition.org/rfp/4834

DOI : $10.4000 / \mathrm{rfp} .4834$

ISSN : 2105-2913

Éditeur

ENS Éditions

Édition imprimée

Date de publication : 30 septembre 2015

Pagination : 61-72

ISSN : 0556-7807

\section{Référence électronique}

Philippe Lemistre, «La professionnalisation des formations initiales : une solution aux mutations du

marché de l'emploi pour les jeunes ? », Revue française de pédagogie [En ligne], 192 I juillet-aoûtseptembre 2015, mis en ligne le 30 septembre 2018, consulté le 03 janvier 2020. URL : http:// journals.openedition.org/rfp/4834; DOI : 10.4000/rfp.4834 


\title{
La professionnalisation des
}

\section{formations initiales : une solution aux mutations du marché de l'emploi pour les jeunes?}

\author{
Philippe Lemistre
}

\begin{abstract}
Cet article resitue la professionnalisation des filières de formation initiale dans le cadre des évolutions du marché du travail, du « régime fordiste » de préprofessionnalisation via la formation initiale au régime financiarisé où la professionnalisation des diplômes du supérieur doit garantir l'employabilité des individus. Le système éducatif anticipe alors, et surtout prend à sa charge publique la régulation des «files d'attente pour l'emploi», avec un gain collectif qui reste à démontrer. Ceci d'autant plus que la sélectivité pour accéder aux diplômes professionnels du supérieur est susceptible de renforcer les inégalités.
\end{abstract}

Mots-clés (TESE) : marché du travail, enseignement supérieur, formation et enseignement professionnels, employabilité, finalités de l'éducation, politique éducative.

La hausse des niveaux d'éducation et la professionnalisation des filières du supérieur semblent idéales pour améliorer l'employabilité des jeunes, mais aussi pour assurer la compétitivité à moyen terme (HCEEE, 2006; Korolitski \& Lichtenberger, 2012).

La professionnalisation emprunte deux voies indissociables pour la formation initiale, celles des contenus et des parcours. Concernant les contenus, la professionnalisation passe par la création de filières professionnelles, comme les licences professionnelles en France en 1999. À la frontière de la professionnalisation des contenus et des parcours, on trouve la mise en situation sur le marché du travail, soit l'apprentissage ou les stages. Viennent ensuite nombre de dispositifs dans le supérieur et aussi en amont, dont l'objectif est de préparer les jeunes pour le marché du travail en facilitant leur orientation dans le système éducatif, en lien avec la réalisation de leur projet professionnel.

Pourquoi un lien si étroit, et particulièrement renforcé dans l'enseignement supérieur, entre formation initiale professionnalisée et marché de l'emploi ? Est-ce parce ce que le système éducatif est à même de répondre aux mutations du marché du travail, grâce aux jeunes et pour les jeunes? Ou est-ce ses mutations 
qui expliquent des exigences renforcées vis-à-vis de l'offre de formation, exigences qui ne sont pas nécessairement favorables aux plus fragiles?

Pour apporter des éléments de réponses à ces questions et comprendre le rôle dévolu aux diplômes des filières professionnelles, il est nécessaire de mettre en relation les évolutions du marché du travail avec le rôle de la formation initiale, que nous limiterons pour cet article au rôle des parcours de formation et des diplômes. En s'appuyant sur les théories économiques dites institutionnalistes, la première partie explique comment, jusqu'aux années 1970, le régime d'accumulation fordiste a fait jouer au diplôme un rôle de préprofessionnalisation. Ce rôle est assez conforme à la représentation du marché du travail proposée par la théorie de la «file d'attente pour l'emploi ». Pour cette dernière, la formation initiale est un moyen pour accéder aux emplois les plus qualifiés, les mieux rémunérés ou les plus favorables à la carrière. Des emplois que l'on trouve au sein des meilleurs segments du marché du travail, selon la terminologie de la théorie de la segmentation du marché du travail. Dans cette perspective et pour cette période, le diplôme est un signal d'adaptabilité à l'emploi pour des entreprises constituées en «marchés internes», qui forment ensuite leur salarié «sur le tas»". La seconde partie explique en quoi, à partir des années 1980, la logique de file d'attente et la segmentation du marché du travail se sont transformées dans le cadre du nouveau régime, dit financiarisé. Les exigences d'adaptabilité immédiate au marché du travail ont eu pour corollaire une sélectivité accrue des parcours, particulièrement pour l'accès aux filières professionnelles du supérieur. La logique de file d'attente pour le marché du travail s'étend alors au système éducatif. Le dernier développement interroge ces évolutions, particulièrement la possible contribution de la professionnalisation à l'augmentation des inégalités, et sa réelle contribution à une amélioration de la situation des jeunes. Plusieurs études que nous avons menées à partir des enquêtes du Centre d'études et de recherches sur les qualifications (Céreq) sont notamment mobilisées à cette fin.

1 Nous nous limiterons à décrire les évolutions en deux régimes d'accumulation pour la France sans y apporter les nuances d'une évidente diversité. Le lecteur peut se reporter à Boyer (2002) pour une synthèse d'ensemble et la mise en exergue de la spécificité du

\section{Le régime fordiste : le diplôme pour se professionnaliser sur le marché du travail}

\section{Jusqu'aux années 1970 : un rôle de préprofessionnalisation pour le diplôme}

De la seconde guerre mondiale aux années 1970, le marché du travail s'insère dans le régime d'accumulation fordiste qui conjugue trois propriétés : une accumulation à dominante intensive sous l'effet de la mécanisation (production «à la chaîne » et consommation de masse), un compromis capital/travail assurant un partage des gains de productivité et un bouclage de l'accumulation principalement au niveau national (Boyer, 2002). Le travail est central, le partage des gains de productivité entre salaires et capital créant un cercle vertueux: les hausses salariales se répercutent sur une hausse de la consommation, donc de la production et des profits, dans une perspective keynésienne (Gaulejac, 2011). La fabrication s'effectue dans une structure hiérarchique et par la division des tâches, souvent répétitives. Le compromis consiste donc principalement à compenser les conditions de travail et la subordination par des augmentations de salaire. Concrètement, au sein de nombreuses entreprises jusqu'au milieu des années 1980, l'augmentation du salaire individuelle ne pouvait résulter que d'une promotion dans un poste plus qualifié ou du changement d'écheIon du salarié en application de règles d'ancienneté dans le poste, et éventuellement de primes de rendement souvent intégrées dans le salaire de base, soit une «régulation conjointe » à partir de l'ancienneté et des postes (Reynaud \& Reynaud, 1994).

Concernant les recrutements, la période débute après guerre par une période de plein emploi et de pénurie de main-d'œuvre. En conséquence, la maind'œuvre est nomade. La rémunération à l'ancienneté a alors pour objectif d'une part de stabiliser les salariés, et de permettre leur formation en interne compte tenu de leur stabilisation d'autre part. II s'agit tout d'abord de qualifier dans l'entreprise des jeunes non diplômés ou de faire évoluer des détenteurs du diplôme professionnel de premier niveau (années 1950-1960). D'où nombre d'individus dotés de bas niveaux de formation qui atteindront les qualifications de techniciens voire de cadre en interne. Le système scolaire avait alors pour fonction de fournir très rapidement des individus formés aux employeurs, avec les bases acquises au plus 
bas niveau (certificat d'études) ou au niveau 5 (CAP), ou des diplômés pourvus de compétences générales pour les niveaux supérieurs. Les employeurs étaient d'autant plus disposés à les doter sur le tas des compétences spécifiques à leurs emplois que les formes d'emploi durable en permettaient l'amortissement. La professionnalisation par la formation initiale s'apparente alors surtout à une préprofessionnalisation.

Toutefois, même si les évolutions de carrières internes sont encore nombreuses, la recherche d'une convergence immédiate entre niveau et compétences acquises en formation initiale et requises dans l'emploi fait son chemin avec plusieurs logiques. Tout d'abord, la massification scolaire avait pour objectif la démocratisation de l'enseignement et l'égalité des chances lors de l'accès au marché du travail. À cette fin, les niveaux d'éducation sont mis en relation avec des niveaux d'emploi. Par exemple est créé en 1959 un diplôme qui contient dans son intitulé la qualification visée : le Brevet de technicien supérieur (BTS). Il s'agissait de favoriser l'égalité des chances d'accéder à la qualification, par l'égalisation des résultats scolaires. Ainsi, la création des CSP (catégories socioprofessionnelles - 1954) visait notamment à fournir une grille d'évaluation des effets de la massification scolaire sur l'égalité des chances (Desrosières \& Thévenot, 2002). Ensuite, la recherche d'une mise en correspondance des formations initiales et des emplois s'opère par la planification, à travers la construction des nomenclatures de formation qui ont aussi pour objectif la prévision des flux de population à scolariser au regard des projections d'emploi par niveau de qualification (Tanguy, 2002). La démarche va s'affiner au fil du temps en s'intéressant davantage aux besoins en spécialités de formation, d'où une professionnalisation des contenus renforcée et une multiplication des filières éducatives et des diplômes.

\section{Professionnalisation et files d'attente pour l'emploi}

La théorie institutionnaliste de la file d'attente aide à comprendre alors quel était et quel est le rôle de la formation initiale (Thurow, 1975). Pour cette théorie, dans la continuité de l'analyse keynésienne, I'hypothèse d'un chômage involontaire est faite. Puisque l'emploi est rationné, il existe des files d'attente pour accéder aux emplois, d'une part. D'autre part, la structure des emplois (la demande) est fixée par des éléments institutionnels dont la régulation ne dépend pas du marché, au sens économique libéral du terme. En clair, pour les institutionnalistes, les salaires, les créations d'emplois, leur répartition selon les qualifications ou les secteurs d'activité ne sont pas la simple résultante d'une confrontation entre offre et demande sur le marché du travail, mais aussi et principalement la conséquence de compromis historiques marqués par les rapports de dominations entre groupes sociaux. Le marché est donc une institution, la représentation du marché proposée par les économistes libéraux est alors une pure fiction théorique éloignée de la réalité, voire au service des classes dominantes (Polanyi, [1946] 1983). Puisque le marché n'est pas concurrentiel, ce n'est pas le travail qui est au centre de l'analyse, mais l'emploi, dont l'accès est moins régulé par les prix que rationné selon des procédures non marchandes. II n'y a pas ici de relation directe entre la combinaison niveau-spécialité et la productivité dans l'emploi, c'est I'adaptabilité plutôt que la productivité qui est recherchée par les employeurs. Ceci distingue très clairement la théorie de Thurow des théories marchandes, dont la théorie du capital humain, contre laquelle il construit son schème théorique. Pour ces dernières, il n'y a pas de problème d'allocation aux emplois, si tant est qu'on laisse faire le marché2.

L'adaptabilité est clairement pour Thurow la capacité à pouvoir être formé pour l'emploi. En effet, les compétences mises en œuvre pour chaque emploi sont supposées procéder en grande partie d'acquis hors formation initiale. "Selon ce modèle, les compétences de la main-d'œuvre n'existent pas sur le marché du travail; au contraire, la plupart des compétences réelles requises dans l'emploi sont acquises de manière informelle par la formation sur le tas» (Thurow, 1972, p. 72). Le niveau de formation est un indice d'adaptabilité à l'emploi qui détermine la place de l'individu dans la file d'attente. Cette perspective correspond bien au rôle des diplômes décrit plus haut pour la période fordiste. Le rôle de la spécialité, et donc de la professionnalisation des contenus, n'est pas envisagé dans cette version initiale. Pour les théories marchandes, la professionnalisation des contenus, soit la spécialisation des formations, conduit uniquement à proposer une nouvelle catégorie de capital humain spécifique à l'emploi. Ce sont les compétences professionnelles (vocational competencies), caractérisées par le fait qu'elles se valorisent sur un sous-ensemble d'emplois (Heijk, Meng \& Ris,

2 Aux problèmes d'information imparfaite près. 
2003)33. Rapporté à la spécialité de formation, cela signifie qu'une «allocation optimale » nécessite de travailler dans sa spécialité.

Toutefois, ce mode d'allocation à l'emploi est considéré comme un cas particulier par la théorie de l'assignment. Cette théorie, à finalité très empirique, s'intéresse à l'allocation des individus aux emplois et tente une synthèse entre les différentes théories économiques appliquées à la formation, tout en privilégiant une approche inspirée de la théorie de la file d'attente (Sattinger, 1993). Dans ce cadre élargi, I'adéquation entre spécialité de formation et d'emploi n'est pas nécessairement, ou du moins pas uniquement, le mode d'allocation le plus optimal. Deux cas de figure sont alors envisageables pour la spécialité. Dans le premier cas, l'emploi est à fort contenu technique, l'adaptabilité à l'emploi est alors étroitement liée aux formations de la spécialité correspondante. Dans le second cas, les contenus de formation de certaines spécialités permettent d'acquérir des compétences qui favorisent l'adaptation à des emplois en dehors de ces spécialités. Ainsi, la hiérarchie des formations pour accéder aux files d'attente pour l'emploi repose autant sur le niveau que sur la spécialité ou la filière (Giret \& Lemistre, 2008). Ceci explique en partie que la correspondance entre spécialité de formation et d'emploi concerne souvent moins de la moitié des diplômés, quels que soient les générations et le niveau en France (Dumartin, 1997; Giret, Lopez \& Rose, 2005), sans que I'on puisse l'expliquer par une inadaptation de l'offre à la demande, et sans que travailler hors de sa spécialité soit nécessairement pénalisant sur le plan salarial (Bruyère \& Lemistre, 2010).

Spécialité et niveau de formation participent donc conjointement de l'adaptabilité des individus aux emplois. Une telle perspective nécessite de s'intéresser aux emplois. Côté emploi, la théorie de la file d'attente s'inscrit dans le courant qui postule une segmentation du marché du travail de Doeringer et Piore (1971)4. D'un côté, des marchés internes, représentés comme des espaces clos, alimentés au bas de la hiérarchie via des postes «ports d'entrée» et permettant des évolutions de carrières en interne (promotions ou salaires). Consi-

3 II s'agit en fait d'une simple acception étendue de la «spécificité", au sens de Becker qui l'appliquait dans son modèle liminaire à la seule formation sur le tas.

4 La théorie de l'assignment se focalise sur l'empirie souvent en examinant l'allocation différenciée davantage en termes de secteurs que d'emplois. Pour la demande, elle revendique uniquement sa dérés comme un tout, ces marchés internes et les salariés concernés constituent le marché primaire. De I'autre côté, les salariés du secteur secondaire ou marché externe, mobiles entre entreprises sans perspective de carrière (Amossé, Péraudin \& Petit, 2011). Jusqu'aux années 1970, le critère de distinction entre marchés internes et externe était l'entreprise, avec un référent privilégié, la taille des entreprises : aux grandes les marchés internes et aux petites le marché externe, les structures de petite taille n'étant pas en mesure de proposer des plans de carrières, car elles n'ont pas un horizon suffisant. À la fin des années 1970, le temps des firmes constituées chacune en un seul et unique marché interne semble révolu. «Ainsi, même dans le secteur le plus primaire des segments du marché du travail, on peut exhiber des différences justifiant une opposition entre deux sous-secteurs, pour peu que l'on détaille les caractéristiques des emplois» (Glaude, 1986, p. 869). Cette évolution correspond à une recherche de flexibilité externe, il s'agit de conserver un volant d'emplois précaires afin d'absorber les chocs conjoncturels. À une segmentation entre entreprises se superpose alors une segmentation entre emplois. Ces évolutions accompagnent la remise en cause du compromis fordiste par les entreprises qui débute après la crise des années 1970. Cette dernière a pour conséquence le souhait d'une baisse des coûts de recrutement et de formation en interne, d'autant plus que les fluctuations du marché s'accentuant, l'horizon temporel des entreprises se raccourcit et permet moins de garantir des carrières longues. De plus, le chômage croissant a rendu caduque la nécessité de la rémunération à l'ancienneté pour stabiliser l'ensemble des salariés, la menace du chômage remplissant cette tâche, sans compter un vieillissement démographique qui rend la rémunération à I'ancienneté coûteuse (Lemistre, 2009). Le souhait et les possibilités de formations en interne s'estompent alors.

\section{Le régime financiarisé : le diplôme professionnel garant de l'employabilité et de l'égalité?}

\section{Le diplôme professionnel pour garantir la qualité de l'emploi}

La recherche de flexibilité et la logique où le travail est davantage un coût qu'un élément essentiel d'un cercle vertueux sont surtout liées à l'entrée dans le nouveau 
mode d'accumulation: le régime d'accumulation financiarisé (Gaulejac, 2011). Au compromis capital/travail se substitue alors la logique de l'actionnaire avec un nouveau cercle vertueux. En visant la rentabilité financière, notamment par la flexibilisation du marché du travail et la modération salariale, les cours de bourse augmenteraient, ce qui permettrait aux ménages de consommer malgré la stagnation de leur salaire, du moins pour ceux qui disposent d'actifs financiers. «La dynamique boursière remplace le salaire comme source de croissance» (Boyer, 2004, p. 93). Disparaît alors un possible antagonisme entre les intérêts des actionnaires et ceux des cadres dirigeants et cadres supérieurs, puisqu'il ne s'agit plus d'arbitrer en faveur du salaire au détriment du profit, stock options et actionnariat salarié faisant converger les intérêts des actionnaires et des managers. On voit ici poindre la conséquence de la logique du nouveau régime d'accumulation : un accroissement des inégalités. En effet, «en régime actionnarial, le risque est porté par les salariés, alors que la masse salariale est considérée comme un coût, une simple variable d'ajustement» (Plihon, 2004, p. 94). Les managers et les actionnaires voient leurs revenus augmenter, alors que les salariés voient leurs conditions se précariser.

L'ensemble de ces constats dessine les contours de l'évolution de la segmentation en France. Dans les années 1980, à la segmentation entre entreprises s'est donc superposée une segmentation entre emplois. L'emploi devient alors central dans l'analyse du marché du travail, y compris pour les institutions européennes qui proposent des comparatifs entre pays selon le critère de "qualité des emplois» (Davoine, Erhel \& Guergoat-Larivière, 2008). La qualité des emplois est évaluée, par exemple, par la stabilité dans l'entreprise (ancienneté dans l'entreprise ou types de contrats, à durée déterminée ou indéterminée), par la proportion de temps partiels subis versus choisis. Le régime financiarisé va renforcer et mettre au premier plan une nouvelle forme de segmentation qui s'ajoute aux deux précédentes : la segmentation entre individus. L'analyse de ce phénomène est liée à la prise en compte du chômage, et plus généralement à une attention plus marquée pour le marché secondaire jusqu'alors négligé dans les analyses de la segmentation du marché du travail (Gazier \& Petit, 2007). Concrètement, pour certains, l'emploi précaire mène à l'emploi précaire à long terme. Segmentations entre emplois et entre individus se superposent alors en tout ou partie (Gazier, 1992). Le repérage statistique de ces trajec- toires instables récurrentes date du début des années 2000. Pour la génération sortie du système éducatif en 1998 enquêtée par le Céreq, 13 \% d'individus se maintiennent au cours des sept premières années de vie active dans l'emploi à durée déterminée, avec des périodes de chômage intermédiaires plus ou moins longues (Couppié, Gasquet \& Lopez, 2007).

Aline Valette (2007) effectue les mêmes constats pour l'ensemble de la population active et propose une analyse dynamique de la segmentation à partir de deux «enquêtes emplois» de I'INSEE. Sont distingués notamment pour le marché primaire : le «marché primaire supérieur» (stabilité dans l'emploi, carrière et hauts salaires) et le «marché primaire inférieur» (stabilité sans carrière). La typologie établie montre l'évolution de la segmentation, et en particulier le recul du marché primaire qui compte $82 \%$ des salariés en 1982 contre $66 \%$ en $2001^{5}$. Cette évolution va de pair avec la mobilité accrue de certains salariés (Amossé, Péraudin \& Petit, 2011).

$\mathrm{Si}$, dans le cadre d'analyses critiques du régime financiarisé (Ramaux, 2006, par exemple), I'augmentation de la mobilité externe comme nécessité pour maintenir la compétitivité internationale est débattue, elle semble faire consensus au niveau des institutions européennes. Ces dernières entendent néanmoins donner une contrepartie à la flexibilité : la sécurisation des parcours, avec notamment pour objectif de limiter la fragilisation des trajectoires et son corollaire, l'accroissement du segment secondaire (Livre vert de 2006, ligne directrice $n^{\circ} 21$ de 2011). Flexibilité et sécurisation des parcours constituent la flexicurité. Elle consiste avant tout à donner les moyens aux individus d'améliorer leur employabilité. «L'employabilité, c'est la capacité à demeurer autonome dans un développement de carrière, la capacité à intéresser successivement plusieurs employeurs, à changer de voie, à forger son itinéraire professionnel» (Gazier, 2003, p. 97), une notion très proche de l'adaptabilité de Thurow.

La sélection par le diplôme est alors amenée à jouer un rôle renforcé, rôle qui lui semble dévolu dès la remise en cause du régime fordiste, car «la décomposition du rapport salarial fordiste à partir de la fin

5 L'auteure montre aussi l'apparition d'une subdivision du marché secondaire en 2001 avec un «marché secondaire stable» avec une ancienneté moyenne élevée (plus de 8 ans), mais des conditions d'emplois dégradées (temps partiel accru, bas salaire), et la subsistance du marché secondaire tel qu'initialement décrit, soit avec des anciennetés dans l'entreprise extrêmement faibles, chacun représentant $17 \%$ des salariés. 
des années 1970 pointe dans la direction d'une différenciation croissante de la relation salariale, c'est-à-dire de la déclinaison du rapport salarial selon les secteurs, les individus ou les entreprises. Quel est dès lors le facteur qui explique la différenciation des trajectoires de salariés tout au long des années 1980 et 1990 ? Dans le cas français, il fait peu de doute que antécédent scolaire et universitaire joue un rôle déterminant » (Boyer, 2003, p. 76).

La professionnalisation des formations initiales a alors pour objectif de permettre à chacun de disposer d'un «signal d'adaptabilité ou d'employabilité » le plus clair possible, ceci pour intégrer le meilleur segment possible du marché du travail et y demeurer, ou limiter les périodes de chômage sur le segment secondaire. La spécialisation et la filière jouent alors un rôle, et la lisibilité du diplôme est essentielle, non seulement pour le premier emploi, mais aussi pour les employeurs potentiels et successifs. À cette fin, tous les diplômes du supérieur ont récemment intégré le fichier du répertoire national des certifications professionnelles. Les diplômes universitaires ont aussi été augmentés du «supplément au diplôme » qui présente le contenu de la formation et les compétences acquises ${ }^{6}$. Récemment, le ministère affiche une nouvelle ambition de «bac - 3 à bac + 3 » pour établir « un continuum de formation articulant les trois années qui précèdent et les trois années qui suivent le baccalauréat» (circulaire MEN-MESR n 2013-2012 du 18 juin 2013). Pour maintenir l'égalité des chances, il faut alors permettre à tous, quel que soit le type de bac notamment, d'accéder à l'enseignement supérieur dans la filière qui leur est la plus adaptée. Pour adapter l'offre à l'ensemble des publics et permettre au plus grand nombre d'accéder à l'enseignement supérieur, la création de filières professionnelles, où les diplômes professionnels se succèdent au fil du cursus, semble essentielle, d'une part. D’autre part, le passage d'une filière générale à une filière professionnalisée et inversement doit être facilité (Korolitski \& Lichtenberger, 2012).

6 Le supplément au diplôme est directement inspiré du dispositif européen Europass, voir notamment la circulaire $n^{\circ}$ 2006-202 du 8 décembre 2006 du ministère de l'Éducation nationale (direc-

\section{Une professionnalisation des parcours élitiste qui socialise les coûts de recrutement?}

Un tel engouement pour la professionnalisation des formations du supérieur est-il légitime? II s'appuie sur l'indéniable constat d'une insertion nettement plus favorable des sortants des filières les plus professionnalisées. Les moyennes cachent néanmoins des disparités internes et entre diplômes et surtout entre individus. Se pose alors la question des inégalités. Car si l'égalité des chances a été un objectif des débuts de la période fordiste et de la massification scolaire, avant de nettement prioriser les objectifs économiques, il n'est pas délaissé. Par exemple, le souhait d'un continuum de bac - 3 à bac +3 est formulé notamment pour contrecarrer la ségrégation associée aux parcours. Dans le registre éducatif, les objectifs liés de la professionnalisation sont la promotion sociale, la remédiation et l'octroi d'une dite "seconde chance» pour les jeunes en difficulté scolaire. Ces jeunes doivent non seulement pouvoir intégrer les filières professionnelles, mais aussi avoir la possibilité d'effectuer des poursuites ou reprises d'études, et ceci, quelle que soit leur origine sociale. Pour la remédiation dans le supérieur, les filières professionnelles semblent la solution pour lutter contre le «décrochage». Le document d'orientation du Plan pluriannuel de réussite en licence ( $P R L, 2007)$ précise que le $P R L$ doit «ouvrir les filières professionnelles (STS, IUT, Licence pro) pour favoriser la réussite de tous les étudiants». Ainsi, la licence professionnelle doit permettre aux jeunes en deuxième année de licence (L2) qui n'ont pas obtenu les résultats nécessaires à la poursuite d'études en filière générale de poursuivre dans une filière professionnelle $e^{7}$. Cette ambition est également soutenue dans le cadre du développement de l'apprentissage qui "offre une seconde chance [...] car il permet de raccrocher des jeunes auxquels le cursus général ne convient pas ${ }^{8}$.

La multiplication des filières et spécialités semble alors parée de toutes les vertus, moyennant une clarification voire une rationalisation des contenus. Toutefois, lorsque Robert Boyer (2003) met en évidence le rôle renforcé de la formation dans la période postfordiste, c'est dans un article où il montre les complémentarités entre la théorie de Bourdieu et la théorie de la

7 Arrêté du 17 novembre 1999.

8 Loi sur l'égalité des chances de 2006 
régulation, quant à l'analyse des conséquences des changements institutionnels. Or, pour la période fordiste, Pierre Bourdieu avait mis en avant les limites de la professionnalisation des contenus, au sens d'une augmentation du nombre de filières professionnelles dans le secondaire, notamment par l'apparition de spécialités. II soulignait le caractère très ségrégué socialement des filières, générales versus professionnelles, ou entre spécialités, les enfants issus des classes populaires étant surreprésentés dans certaines filières (Bourdieu, 1979). Cette analyse des années 1970 appliquée à l'enseignement secondaire a été transposée, ou plutôt étendue, à l'enseignement supérieur pour la période postfordiste. C'est la démocratisation ségrégative (Merle, 2012). Cette notion met en évidence ce que peut masquer la démocratisation quantitative ou supposée uniforme, au sens d'une augmentation généralisée des niveaux d'éducation qui en réalité masque des logiques internes à chaque niveau, logiques qui participent de la reproduction sociale. Cette dernière ne passe plus seulement alors par le niveau de formation, mais de plus en plus par la filière.

Le niveau continue néanmoins à jouer un rôle de plus en plus discriminant. Toutes les études citées sur la segmentation convergent, dès lors que le diplôme est pris en compte : plus le diplôme est élevé plus l'accès au marché primaire est probable. Une tendance qui s'est nettement renforcée, par exemple entre 1982 et 2001, pour l'accès au marché primaire supérieur (Valette, 2007). Dans le cadre de la démocratisation ségrégative, la hiérarchie des diplômes n'est pas seulement celle des niveaux. Au sein de chaque niveau, les filières et les spécialités sous-tendent une hiérarchie des diplômes sur le marché du travail (Dauty, Lemistre \& Vincens, 2006), conformément à I'hypothèse de la théorie de la file d'attente. De plus, actuellement, les jeunes sont de plus en plus diplômés et recrutés sur des premiers emplois «déclassés " (Monso, 2006). Or, le déclassement est très différencié selon la filière et la spécialité de diplôme ${ }^{9}$. Par exemple, trois ans après la sortie du système éducatif à bac $+2,57 \%$ des jeunes sortants de la filière «secrétariat et bureautique» n'accèdent pas à une qualification au moins

9 Le déclassement est une notion polysémique. Analysé par rapport au diplôme, la mesure la plus simple usitée pour les références citées, qui peut être qualifiée «d'institutionnelle», c'est le niveau de qualification auquel devrait donner accès un diplôme selon l'institution. Par exemple, un détenteur d'un diplôme de niveau bac +5 est censé occuper un emploi de cadre, le détenteur d'un Brevet de technicien supérieur un emploi de niveau technicien, etc. égale à profession intermédiaire (dont technicien, agent de maîtrise), contre $18 \%$ pour «informatique», et $34 \%$ pour les diplômés de licence de la spécialité «comptabilité, gestion $»^{10}$. Surtout, la hausse des niveaux d'éducation transforme la logique de file d'attente dans certaines filières. Par exemple, dans le domaine "échange et gestion », trois ans après la sortie du système éducatif de la génération de sortants de 1998 à la génération 2007, la proportion de jeunes qui n'accèdent pas à l'emploi-cadre augmente pour les diplômés de masters et d'écoles de commerce de respectivement $36 \%$ à $46 \%$ et $17 \%$ à $37 \%$. Soit un effet tant de l'augmentation généralisée des niveaux d'éducation que de la multiplication sur la période des écoles de commerce au sens large ${ }^{11}$. L'écart qui s'amenuise, mais se maintient, entre écoles de commerce et masters, est notamment lié à la démocratisation ségrégative. En effet, pour les générations étudiées, la proportion d'enfants de pères cadres est supérieure de plus de $20 \%$ dans les écoles de commerce à celle des masters (Lemistre, 2013).

Les effets de la démocratisation ségrégative sont néanmoins surtout étudiés en regard des parcours dans le système éducatif. Il est possible alors d'illustrer des logiques de file d'attente qui prennent leur source dans le système éducatif avant de se prolonger vers le marché du travail. Dans ce domaine, l'exemple le plus récent de professionnalisation des contenus est la création de la licence professionnelle dotée d'une filière apprentissage. L'enquête du Céreq qui permet d'observer la génération sortie du système éducatif en 2007 en emploi en 2010 illustre a priori l'indéniable supériorité de la filière professionnelle (Kergoat \& Lemistre, 2014). Par rapport aux sortants diplômés de licence générale sans poursuite d'études ${ }^{12}$, les salaires moyens des diplômés de licence professionnelle sont supérieurs d'un tiers, et de $10 \%$ de plus pour ceux qui ont effectué leur licence professionnelle par apprentissage. Les constats sont similaires pour l'accès à l'emploi qualifié : plus le degré de professionnalisation augmente, plus l'insertion est favorable. Toutefois, ce

10 Résultats pour trois enquêtes Céreq agrégées de 2001, 2004, 2007 (générations de sortants de 1998, 2001 et 2004 observés trois ans plus tard). À noter que les logiques de spécialité ne recouvrent pas essentiellement les logiques de genre (Lemistre, 2013). 11 Intégrant les écoles privées de gestion non recensées parmi les écoles classées.

12 Un jeune sur dix quitte la licence générale sans poursuite d'études, ce qui représente un effectif in fine assez proche de celui des sortants de licences professionnelles pour la génération 2007. 
constat doit être relativisé. En effet, les écarts s'estompent fortement dès lors que I'on neutralise les effets du parcours antérieur et de la spécialité ${ }^{13}$. Par exemple, la détention d'un DUT ou d'un BTS est un atout majeur à la faveur des licences professionnelles dont $80 \%$ des sortants sont issus, contre $20 \%$ pour les licences générales. En amont, la majorité de ces diplômés de licence professionnelle ayant un parcours BTSDUT sont issus de bacs généraux, et les autres parmi les plus performants en filière technologique. La proportion de bacs généraux parmi les étudiants qui accèdent à la licence professionnelle après la L2 universitaire est encore plus élevée (neuf sur dix). Par ailleurs, parmi les sortants de licences, les détenteurs de licences professionnelles sont surreprésentés dans les domaines de spécialité de formation et dans les secteurs d'activités professionnelles qui offrent la meilleure insertion, que l'on sorte de licence professionnelle ou générale ${ }^{14}$. En résumé, la plus-value à l'insertion associée à la licence professionnelle est en quasi-totalité associée à un effet de sélectivité des publics de licences professionnelles et aux choix d'ouvrir ces filières dans les domaines où les jeunes étaient déjà les plus employables.

Cela ne signifie pas que la formation associée au diplôme professionnel de fin de parcours, en soi, n'apporte pas de compétences complémentaires, mais plutôt qu'elle le fait pour les individus déjà les plus employables, au vu de leurs choix de spécialités et de leurs parcours antérieurs, soit parce qu'ils sont déjà détenteurs de diplômes professionnels, soit parce qu'ils figurent parmi les plus performants au baccalauréat. Il n'y a donc pas de seconde chance pour les premières années de licence de filières professionnelles en difficulté dans le supérieur, ou dont les résultats au bac étaient insuffisants, sans le moindre doute une population majoritairement de milieu social modeste. Pour autant, la filière professionnelle, au sens de la succession de diplômes professionnels, existe, mais pas pour une majorité de jeunes, puisque même si les DUT-BTS sont majoritaires en licence professionnelle, les bacs pros et technos ne le sont pas. En d'autres termes, les jeunes d'origine sociale modeste, qui empruntent davantage les voies professionnelles, par-

13 Reste un écart moyen de salaire de moins de $10 \%$ entre les licences professionnelles et générales.

14 Si les caractéristiques de l'emploi (secteur d'activité, quotité de travail, $C D I, C D D$ ) sont prises en compte dans le modèle toutes choses égales par ailleurs, la plus-value des licences professionnelles ticulièrement pour les bacs technos ou pros ${ }^{15}$, bénéficient de cette filière à la condition d'être très performants. À noter que la logique de sélectivité se poursuit maintenant au-delà de la licence pro. Alors qu'avant même la création des licences professionnelles en 1999, $80 \%$ des détenteurs de DUT poursuivaient leurs études, déjà $20 \%$ des diplômés de licence professionnelle le faisaient moins de 10 ans après sa création (Borras, Epiphane, Lemistre et al., 2012), sans surprise puisque ce sont des étudiants sur-sélectionnés!

Encore peut-on identifier une filière pour certains : du bac technologique à la licence professionnelle, après un BTS ou un DUT. Ce n'est pas le cas pour l'apprentissage où seulement un apprenti sur dix du supérieur possède un diplôme obtenu par apprentissage à un niveau inférieur. Ainsi, il n'y a pas de «filière apprentissage», car les apprentis du haut ne sont pas les apprentis du bas (Kergoat \& Lemistre, 2014). Plus encore, la sélection des apprentis dans le supérieur semble étroitement liée aux dispositions culturelles et sociales des candidats, sont ainsi avantagés les candidats bénéficiant d'une solide formation générale en amont ou d'une socialisation familiale ad hoc, soit les jeunes de milieux relativement favorisés parmi les candidats à l'apprentissage (Kergoat, 2011). On retrouve ces logiques de sélectivité sociale des parcours pour la plupart des parcours du supérieur. Par exemple, pour la dernière génération (2010) enquêtée en 2013 par le Céreq, les jeunes dont les deux parents ont un niveau d'études inférieur au bac sont représentés dans les proportions suivantes pour chaque parcours : «bac pro puis BTS-DUT » versus «bac général puis BTS-DUT» respectivement $61 \%$ et $42 \%$; «bac pro ou techno puis BTS-DUT puis licence pro» versus «bac général puis BTS-DUT puis licence pro », $45 \%$ et $36 \%$; «au moins un diplôme professionnel puis diplôme d'ingénieur » versus «intégralité du parcours antérieur en filières générales puis diplôme d'ingénieur», $40 \%$ contre 18\% (Lemistre, 2015) ${ }^{16}$. Sans compter les nouvelles stratégies de double diplomation au niveau master, particulièrement développées pour les jeunes des milieux sociaux favorisés (Borras, Epiphane, Lemistre et al.,

$155 \%$ des détenteurs d'un DUT-BTS avec un bac techno ont un père ou une mère qui possède un diplôme de niveau bac +5 au moins, $15 \%$ parmi les détenteurs d'un DUT-BTS possèdent un bac général, par exemple (source : enquête Céreq 2013 ; Lemistre, 2015) 16 Autres exemples: «DUT-BTS puis master 2 » versus «parcours général (de la $3^{\mathrm{e}}$ à la licence) puis master $2 », 36 \%$ contre $28 \%$; «au moins un diplôme professionnel puis école de commerce» versus "parcours général puis école de commerce», $32 \%$ contre $18 \%$. 
2012 ${ }^{17}$. La sélectivité des files d'attente dans le système éducatif qui déterminent en grande partie la place dans les files d'attente pour l'emploi est donc socialement très marquée.

\section{Conclusion}

Nous avons tenté de resituer dans cet article la professionnalisation des filières dans le cadre des évolutions du marché du travail, ceci en distinguant deux périodes dans la perspective de la théorie de la régulation : un régime fordiste, puis un régime financiarisé. Dans le premier, le travail est au cœur du compromis fordiste, le système éducatif participe alors plutôt d'une préprofessionnalisation. C'est la formation sur le tas qui assure la professionnalisation des individus. La concurrence accrue et son corollaire, le passage au régime financiarisé, changent la donne. Ce n'est plus le travail qui prime, mais l'actionnaire, le travail est un coût qui doit être minimisé. Ces évolutions ont une conséquence importante sur la segmentation du marché du travail qui sépare salariés stables ou "à carrière » des autres. Alors qu'elle s'effectuait globalement entre entreprises (grandes versus petites), à partir des années 1980, elle s'est effectuée davantage entre emplois, avec des salariés stables et précaires coexistant dans les mêmes entreprises. Dans les années 2000, un nouveau mode de segmentation se superpose aux deux précédents : la segmentation entre individus. L'emploi précaire succède à l'emploi précaire à long terme pour certains individus. Pour caractériser les trajectoires individuelles, la référence à la qualité de l'emploi (carrière, stabilité, rémunération, qualification, etc.) prend alors un nouvel essor relayé au niveau européen.

Le rôle du diplôme se renforce alors dans la logique de la «file d'attente» pour l'emploi, que nous avons abordée via la théorie non marchande du même nom (théorie de la file d'attente). II s'agit de se doter des meilleurs signaux d'adaptabilité (ou d'employabilité) afin d'accéder aux emplois de "qualité». Dans cette perspective, la professionnalisation des filières et des diplômes joue alors un rôle fondamental, au moins du point de vue des institutions. Ces dernières entendent doter les individus des compétences les plus adéquates aux domaines d'emploi visés, tout en aidant les jeunes à s'orienter et à formuler leur projet professionnel. La lisibilité du diplôme est aussi devenue essen-

17 Un des deux parents a au moins un bac +5 pour les jeunes qui ont un parcours général puis deux masters (Lemistre, 2015). tielle, car les individus sont censés être de plus en plus mobiles entre entreprises. Une certification où les compétences acquises sont bien circonscrites et identifiées pour les employeurs participe alors de la sécurisation des parcours, sécurisation qui est une contrepartie d'une flexibilité accrue, la combinatoire donnant l'actuelle logique de «flexicurité».

À l'appui de cette hypothèse est mise en avant la meilleure insertion des diplômés des filières professionnelles du supérieur en France. De fait, les détenteurs de diplômes professionnels s'insèrent mieux que les autres, particulièrement pour le supérieur, comme le montrent nombre d'études et quelques résultats produits ici, notamment pour la dernière certification créée : la licence professionnelle. Mais la professionnalisation a un coût, qui n'est autre que le transfert vers la collectivité d'une part des coûts de recrutement et de formation jusqu'alors assumés par les entreprises, en particulier dans le contexte du régime fordiste. Ce serait le prix à payer pour, d'une part, rester compétitif au niveau international, grâce à une main-d'œuvre mieux dotée en compétences à la sortie du système éducatif, et pour la sécurisation des trajectoires individuelles, d'autre part. Pour ce dernier aspect, encore faut-il que la professionnalisation profite aux individus susceptibles d'être les plus fragilisés sur le marché du travail : les jeunes les moins performants dans le système éducatif, et aussi ceux d'origine sociale modeste, notamment pour leur permettre d'accéder à l'enseignement supérieur où ils sont nettement sousreprésentés. Or, les résultats de plusieurs études produites ici suggèrent que ce n'est pas le cas. L'accès aux filières professionnelles du supérieur est réservé en majorité aux jeunes les plus dotés en capital social. Quant aux autres, s'ils sont issus de milieux sociaux plus modestes, ils ne peuvent prétendre à ces filières qu'à la condition sine qua non d'un parcours antérieur où les performances scolaires étaient suffisantes, notamment pour accéder à des filières sélectives en amont. La logique est donc méritocratique, mais fortement biaisée par des logiques sociales où se déploient à nouveau les stratégies de classe.

La «professionnalisation» de l'enseignement supérieur associée au souhait d'une hausse des niveaux d'éducation en France, loin d'atteindre son ambition affichée de promouvoir la «deuxième chance» et de favoriser la promotion sociale, contribuerait alors à renforcer, déplacer, voire légitimer des inégalités d'accès aux emplois qui s'accentuent dans le régime financiarisé. Ceci alors que la plus-value constatée à 
I'insertion pour ces formations pourrait être en partie le résultat d'une sélectivité des publics potentiellement les plus employables. En créant de nouvelles logiques de files d'attente dans le système éducatif, ce dernier anticipe alors et surtout prend à sa charge «publique » la régulation des files d'attente pour l'emploi, avec un gain collectif qui, lui, reste à démontrer.

Philippe Lemistre

CÉREQ-CERTOP, Université Toulouse Jean-Jaurès et CNRS philippe.lemistre@univ-tlse2.fr

\section{Bibliographie}

AMOSSÉ T., PÉRAUDIN C. \& PETIT H. (2011). « Mobilité et segmentation du marché du travail : quel parcours professionnel après avoir perdu ou quitté son emploi ? ». Économie et statistiques, $\mathrm{n}^{\circ} 450$, p.79-105.

BORRAS I., EPIPHANE D., LEMISTRE P. \& RIK G. (dir.) (2012). «Étudier en Licence : parcours et insertion». Relief, $\mathrm{n}^{\circ} 36$.

BOURDIEU P. (1979). La Distinction. Paris : Éd. de Minuit.

BOYER R. (2002). «Variété du capitalisme et théorie de la régulation ». In R. Boyer \& Y. Saillard, Théorie de la régulation état des savoirs. Paris : La Découverte, p. 125-195.

BOYER R. (2003). «L'anthropologie économique de Pierre Bourdieu ». Actes de la recherche en sciences sociales, vol.5, $\mathrm{n}^{\circ} 150$, p. $65-78$

BOYER R. (2004). Théorie de la régulation. Paris : La Découverte.

BRUYÈRE M. \& LEMISTRE P. (2010). «La formation professionnelle des jeunes : quelle valorisation de la spécialité?». Revue d'économie politique, $\mathrm{n}^{\circ} 120(3)$, p. 539-561.

COUPPIÉ T., GASQUET C. \& LOPEZ A. (2007). Quand la carrière commence... Les sept premières années de vie active de la Génération 98. Marseille : Céreq.

DAUTY F., LEMISTRE P. \& VINCENS J. (2006). «Le sens, la portée et le devenir de la nomenclature des niveaux de formation ». CPC document, $\mathrm{n}^{\circ} 2006 / 2$.

DAVOINE L., ERHEL C. \& GUERGOAT-LARIVIÈRE M. (2008). «Monitoring Employment Quality in Europe: European Employment Strategy Indicators and Beyond». International Labour Review, vol.147, n²-3, p.163-198.

DESROSIÈRES A. \& THÉVENOT L. (2002). Les catégories socioprofessionnelles. Paris : La Découverte.

DOERINGER P. \& PIORE M. (1971). Internal labor markets and manpower analysis. Lexington (États-Unis) : Heath Lexington books.

DUMARTIN S. (1997). «Formation-emploi, quelle adéquation?». Économie et statistique, n³03, p.59-79.

GAULEJAC V. (2011). Travail, les raisons de la colère. Paris : Éd. du Seuil.

GAZIER B. (1992). Économie du travail et de l'emploi. Paris : Dalloz.

GAZIER B. (2003). Tous sublimes. Vers un nouveau plein emploi. Paris : Flammarion.

GAZIER B. \& PETIT H. (2007). «French Labour Market Segmentation and French Labour Market Policies since the Seventies: Connecting Changes». Économies et sociétés, série Économie du travail, n²8, p.1027-1056.

GIRET J.-F. \& LEMISTRE P. (2008). «Les théories du filtre et du signalement». In J.-J. Paul \& J. Rose, Le tour des relations formation-emploi-travail en cinquante-cinq questions. Paris : Dunod, p.139-144.

GIRET J.-F., LOPEZ A. \& ROSE J. (2005). Des formations pour quels emplois? Paris : La Découverte.

GLAUDE M. (1986). «Ancienneté, expérience et théorie dualiste du marché du travail». Économie appliquée, n4, p. 847-876.

HCEEE (2006). «Objectif 50\% d'une génération diplômée de l'enseignement supérieur». Rapport du Haut comité éducation-économie-emploi.

HEIJK H., MENG C. \& RIS C. (2003). «Fitting to the Job: The Role of Generic and Vocational Competencies in Adjustment and Performance». Labour Economics, n 10, p. 215-219.

KERGOAT P. (2011). «Quand les grandes entreprises définissent les modalités d'accès à l'éducation ». Éducation permanente, hors série AFPA, p. 89-104.

KERGOAT P. \& LEMISTRE P. (2014). «Professionnalisation de l'enseignement supérieur et sélection des publics : les faux-semblants de la démocratisation ». Économies et sociétés, série Économie du travail, n³6, p. 1405-1438.

KOROLITSKI J.-P. \& LICHTENBERGER Y. (2012), «Quelle valeur accorder à la licence, pour quels étudiants?». Administration et éducation, $\mathrm{n}^{\circ} 1, \mathrm{p}$.105-110.

LEMISTRE P. (2003). «Transformation des marchés internes et emplois en France ». Économie appliquée, n² 2, p. 123-160.

LEMISTRE P. (2009). «Rémunération à l'ancienneté et individualisation des salaires : actualité de la théorie du paiement différé ». Économies et sociétés, série Économie du travail, $n^{\circ} 23$, p. 67-88.

LEMISTRE P. (2013). «Le déclassement des sortants du supérieur : une affaire de spécialité, de niveau ou d'individu?». Relief, $\mathrm{n}^{\circ} 42$, p. 117-131.

LEMISTRE P. (2015). «De bac moins 3 à bac plus 3 : le sens de la professionnalisation des filières, au vu des parcours et de l'insertion ». Relief, $n^{\circ} 50$.

MERLE P. (2012). La ségrégation scolaire. Paris : La Découverte.

MONSO O. (2006). «Changer de groupe social en cours de carrière. Davantage de mobilité depuis les années quatrevingt ». Insee Première, $\mathrm{n}^{\circ} 1112$.

PETIT H. (2002). Fondements et dynamique de la segmentation du marché du travail- une analyse sur données françaises. Thèse de doctorat, sciences économiques, université de Paris I. 
PLIHON. D. (2004). Le nouveau capitalisme. Paris : La Découverte

POLANYI K. ([1946] 1983). La grande transformation. Paris : Gallimard.

RAMAUX C. (2006). Emploi : éloge de la stabilité. L'État social contre la flexicurité. Paris : Fayard.

REYNAUD E. \& REYNAUD J.-D. (1994). «La régulation conjointe et ses dérèglements ». Le Travail humain, vol.57, $\mathrm{n}^{\circ} 3$, p.227-238.

SATTINGER M. (1993). «Assignment models of the distribution of earnings ». Journal of Economic Literature, $\mathrm{n}^{\circ} 31$, p. $831-880$.
TANGUY L. (2002). «La mise en équivalence de la formation avec l'emploi dans les IV et Ve Plans (1962-1970)». Revue française de sociologie, $\mathrm{n}^{\circ} 43-4$, p. 685-709.

THUROW L. C. (1972). «Education and Economic Equality». The Public Interest, $n^{\circ} 28$, p. 66-81.

THUROW L. C. (1975). Generating inequality. New York : Basic Books.

VALETTE A. (2007). «Systèmes d'emploi français et britannique : évolutions entre 1980 et 2001 ». Économies et sociétés, série Économie du travail, n²29, p. 1925-1958. 
